

\title{
Fiber-optic probe for noninvasive real-time determination of tissue optical properties at multiple wavelengths
}

\author{
Jan S. Dam, Carsten B. Pedersen, Torben Dalgaard, Paul Erik Fabricius, \\ Prakasa Aruna, and Stefan Andersson-Engels
}

\begin{abstract}
We present a compact, fast, and versatile fiber-optic probe system for real-time determination of tissue optical properties from spatially resolved continuous-wave diffuse reflectance measurements. The system collects one set of reflectance data from six source-detector distances at four arbitrary wavelengths with a maximum overall sampling rate of $100 \mathrm{~Hz}$. Multivariate calibration techniques based on twodimensional polynomial fitting are employed to extract and display the absorption and reduced scattering coefficients in real-time mode. The four wavelengths of the current configuration are $660,785,805$, and $974 \mathrm{~nm}$, respectively. Cross-validation tests on a $6 \times 7$ calibration matrix of Intralipid-dye phantoms showed that the mean prediction error at, e.g., $785 \mathrm{~nm}$ was $2.8 \%$ for the absorption coefficient and $1.3 \%$ for the reduced scattering coefficient. The errors are relative to the range of the optical properties of the phantoms at $785 \mathrm{~nm}$, which were $0-0.3 / \mathrm{cm}$ for the absorption coefficient and $6-16 / \mathrm{cm}$ for the reduced scattering coefficient. Finally, we also present and discuss results from preliminary skin tissue measurements. (c) 2001 Optical Society of America
\end{abstract}

OCIS codes: $170.1470,170.1580,170.3890,170.6510$.

\section{Introduction}

The optical properties of human tissue, ${ }^{1}$ i.e., the absorption coefficient $\mu_{a}$, the scattering coefficient $\mu_{s}$, and the anisotropy factor $g$, may provide important information on the composition and the physiological dynamics of the tissue. Whereas $\mu_{a}$ may provide information on tissue chromophores, ${ }^{2-4} \mu_{s}$ and $g$ may be used to characterize the form, size, and concentration of various scattering components in the tissue. ${ }^{5-8}$

Owing to the obvious advantages of noninvasive and minimally invasive measurements, determination of tissue optical properties based on diffuse reflectance measurements has a significant potential in biomedical diagnostics and monitoring. Diffuse reflectance measurements may be roughly divided into time-resolved $^{9,10}$ (TR), frequency-domain ${ }^{11,12}$ (FD),

J. S. Dam (jan.sorensen_dam@fysik.lth.se), C. B. Pedersen, T. Dalgaard, and P. E. Fabricius are with Bang \& Olufsen Medicom a/s, DK-7600, Struer, Denmark. S. Andersson-Engels is with the Department of Physics, Lund Institute of Technology, P.O. Box 118, SE-22100, Lund, Sweden. P. Aruna is with Anna University, 600025 Madras, India.

Received 26 May 2000; revised manuscript received 7 December 2000.

0003-6935/01/071155-10\$15.00/0

(C) 2001 Optical Society of America and spatially resolved continuous-wave ${ }^{13-21}$ (cw) methods. Traditionally, TR- and FD-based methods have been considered to be more accurate for absolute determination of optical properties than cw based methods. However, TR and FD methods also require more bulky and expensive equipment, and a larger sample volume, which may restrict some biomedical applications, e.g., implementation in portable monitoring equipment or in endoscopes and catheters. Recent research ${ }^{19}$ has shown that cwbased methods may yield absolute determination of the optical properties of tissue with accuracies similar to the TR and FD methods, which makes cw-based methods a better choice for many practical applications.

Continuous-wave diffuse reflectance methods may be further divided into methods based on probes in contact with the tissue and noncontact methods, i.e., image reflectometry. The latter method is advantageous in clinical applications because of the noncontact and thus sterile properties. The advantage of contact probes is that they can be made small and portable. Therefore they are well suited for (a) optical biopsies of body cavities or organs, because a probe may be implemented in existing endoscopic equipment, or (b) for long-time monitoring of tissue optical properties, because the probe may be fixed to the skin and still allow the patient to move around. 


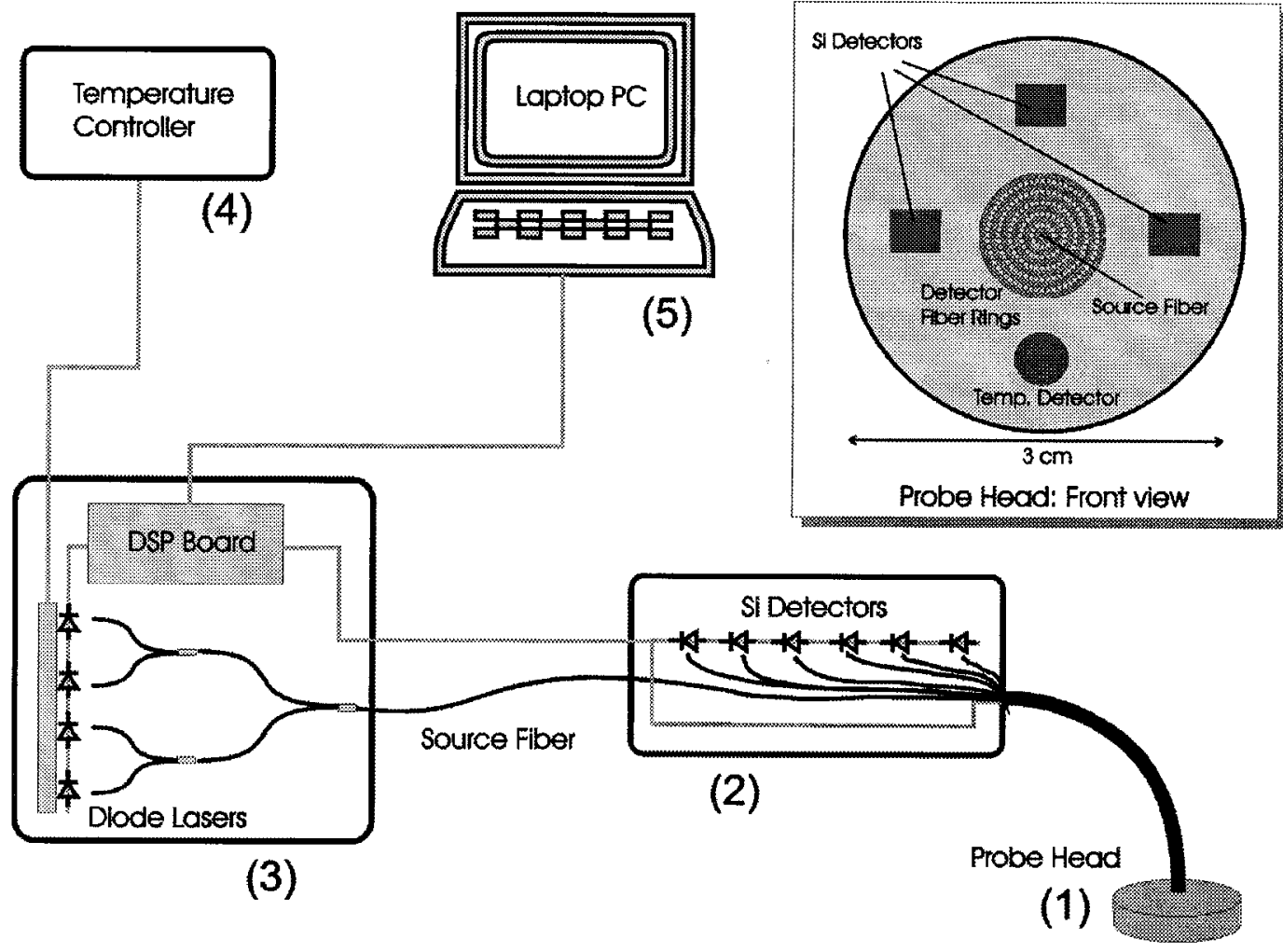

Fig. 1. Diagram of the fiber-optic system for $R(r)$ measurements applied in this paper. (1) Probe head with source and detector optical fibers mounted in a rotational symmetric configuration. (2) Handheld box with silicon photodiodes and amplifier electronics. (3) Stationary box containing a digital signal processing (DSP) board and the light sources in the form of diode lasers. (4) External temperature controller to maintain a constant temperature of the diode lasers. (5) Laptop PC to analyze, display, and store the acquired $R(r)$ data.

However, there are a number of drawbacks related to skin tissue monitoring using a contact probe system. First, the static mechanical pressure of the probe may influence the tissue optical properties, e.g., by displacement of blood in the sample volume. Second, physical activities of the patient may lead to motion artifacts, i.e., movements of the probe relative to the skin tissue. Finally, obstruction of the tissue and sweating may cause long-term changes in the tissue optical properties and the probe-skin optical interface. All such contributions have to be isolated to extract the relevant information from diffuse reflectance measurements.

Accurate closed-form mathematical analytical expressions for spatially resolved diffuse $\mathrm{cw}$ reflectance $R(r)$ as a function of the optical properties are strongly limited by requirements to the range of the optical properties and the specific geometry of the setup. ${ }^{22}$ Therefore methods based on more-accurate numerical light-propagation models, e.g., Monte Carlo simulations, ${ }^{23}$ are often used in conjunction with various forms of multivariate analysis ${ }^{19,24}$ to solve the inverse problem of extracting the optical properties from $R(r)$ measurements. However, as a consequence of the similarity principle, ${ }^{25,26}$ normally only $\mu_{a}$ and the reduced scattering coefficient $\mu_{s}{ }^{\prime}=$ $(1-g) \mu_{s}$ are extracted from $R(r)$ measurements.
In this paper we present a fiber-optic probe system for in vivo real-time determination of tissue optical properties based on $R(r)$ measurements at four arbitrary wavelengths. The probe system collects the diffuse reflectance at six distances, extracts the corresponding $\mu_{a}$ and $\mu_{s}{ }^{\prime}$, and displays the results immediately on a laptop personal computer (PC). The prediction of $\mu_{a}$ and $\mu_{s}^{\prime}$ is based on multiple polynomial regression ${ }^{27}$ and calibration on a set of Intralipid-dye phantoms with well-known optical properties within a range typical for biologic tissue ${ }^{28}$ in the visible and the near-infrared regions. In the following sections we first give a description of the probe specifications and the principles of the calibration and prediction algorithms. Next, we present and discuss the results obtained from phantom measurements and simulated numerical tests with respect to prediction errors, robustness, and the like. Finally, we present the results of preliminary prediction tests obtained from a series of clinical measurements on normal skin tissue.

\section{Materials and Methods}

\section{A. Fiber Probe System}

Figure 1 shows the basics of the fiber probe system we used for the measurements described below. The 
system consists of a probe head with a $200-\mu \mathrm{m}$ source fiber in the center surrounded by five equally spaced concentric rings of $250-\mu \mathrm{m}$ detector fibers. We chose this ring geometry instead of, e.g., a simpler linear geometric configuration, in part to be able to collect more light at farther distances and in part to minimize any problems arising from tissue inhomogeneities during clinical measurements. The fibers of each single ring detector are bundled and terminated on separate silicon photodiodes. In addition, three photodiodes and a temperature sensor are mounted directly near the perimeter of the probe head. Thus $R(r)$ can be collected at six distances, in part at the five fiber rings $(r=0.6,1.2,1.8,2.4$, and $3.0 \mathrm{~mm})$ and in part at the directly mounted photodiodes $(r=7.8$ $\mathrm{mm})$. These distances were chosen on the basis of previous studies. ${ }^{19}$ The gain of each reflectance detector was calibrated in an integrating sphere setup to obtain equal outputs at constant input light intensities. The source fiber is coupled into four separate fibers, each connected to four replaceable low-power diode lasers. The diode lasers are mounted on a heat sink with a constant temperature maintained by an external controller. Furthermore, a separate reference detector monitors the output of the source fiber at the probe head. The diode lasers may be selected arbitrarily to suit different applications. In this paper we used diode lasers with the wavelengths $660,785,805$, and $974 \mathrm{~nm}$, which are well suited for applications involving hemodynamic monitoring. The data acquisition and storage is controlled by a laptop PC connected to a digital signal processing (DSP) board. In each $R(r)$ measurement the detector hardware collects data simultaneously in eight parallel channels from the probe head, i.e., from the six detector rings, from the reference detector at the source fiber, and from the temperature sensor. One cycle of four successive measurements (i.e., one at each wavelength) including dark measurements may be performed in $\sim 10 \mathrm{~ms}$; thus the maximum sampling rate of the system is $\sim 100 \mathrm{~Hz}$. To minimize any interference from background light or drift of the light source, the dark measurements are subtracted from the measured reflectance data after which they are normalized relative to the source reference. The DSP board accomplishes this prior to when the data are analyzed, displayed, and stored by the PC.

\section{B. Calibration and Prediction Algorithms}

In theory, $\mu_{a}$ and $\mu_{s}{ }^{\prime}$ may be determined with $R(r)$ data from only two of the six detector distances of the fiber probe. Building on our previous research, ${ }^{19,27}$ we thus applied multiple polynomial regression (MPR) to create a calibration model and subsequently extract $\mu_{a}$ and $\mu_{s}{ }^{\prime}$ from $R(r)$ measurements at $r_{1}=0.6$ $\mathrm{mm}$ and at $r_{2}=7.8 \mathrm{~mm}$. For the sake of clarity we give a summary of the MPR method here. We first measure $R(r)$ at $r_{1}$ and at $r_{2}$ for a set of calibration samples with well-defined optical properties and de- note them $R_{1, \text { cal }}$ and $R_{2, \mathrm{cal}}$. Then we find a doublepolynomial fit to $R_{1, \text { cal }}$ and $R_{2, \text { cal }}$ :

$$
\begin{aligned}
R_{1, \mathrm{fit}}\left(\mu_{a}, \mu_{s}{ }^{\prime}, m\right)= & \left(a_{0}+a_{1} \mu_{a}+a_{2} \mu_{a}{ }^{2}+\ldots a_{m} \mu_{a}{ }^{m}\right) \\
& \times\left(b_{0}+b_{1} \mu_{s}{ }^{2}+\ldots b_{m} \mu_{s}{ }^{\prime m}\right), \\
R_{2, \mathrm{fit}}\left(\mu_{a}, \mu_{s}{ }^{\prime}, m\right)= & \left(c_{0}+c_{1} \mu_{a}+c_{2} \mu_{a}{ }^{2}+\ldots c_{m} \mu_{a}{ }^{m}\right) \\
& \times\left(d_{0}+d_{1} \mu_{s}{ }^{2}+\ldots d_{m} \mu_{s}{ }^{\prime m}\right),
\end{aligned}
$$

Where $a, b, c$, and $d$ are fitting coefficients determined by least-squares regression. $R_{1, \text { fit }}$ and $R_{2, \text { fit }}$ thus constitute the calibration model. The next step is to solve the inverse problem of determining $\mu_{a}$ and $\mu_{s}{ }^{\prime}$ from $R(r)$ measurements on a set of prediction samples, i.e., $R_{1 \text {,meas }}$ and $R_{2 \text {,meas }}$. First we define

$$
\begin{aligned}
& F\left(\mu_{a}, \mu_{s}{ }^{\prime}\right)=R_{1, \text { fit }}-R_{1, \text { meas }}, \\
& G\left(\mu_{a}, \mu_{s}{ }^{\prime}\right)=R_{2, \text { fit }}-R_{2, \text { meas }} .
\end{aligned}
$$

Then we use a Newton-Raphson algorithm to perform converging iterative calculations of $\mu_{a}$ and $\mu_{s}{ }^{\prime}$ :

$$
\begin{aligned}
& \left.-\left[\begin{array}{c}
\mathbf{F}\left(\mu_{a, k}, \mu_{s, k}{ }^{\prime}\right) \\
\mathbf{G}\left(\mu_{a, k}, \mu_{s, k}^{\prime}\right)
\end{array}\right]=\left[\begin{array}{cc}
\frac{\partial F}{\partial \mu_{a}} & \frac{\partial F}{\partial \mu_{s}^{\prime}} \\
\frac{\partial G}{\partial \mu_{a}} & \frac{\partial G}{\partial \mu_{s}^{\prime}}
\end{array}\right]\left(\begin{array}{c}
h_{a, k} \\
h_{s, k}
\end{array}\right)\right\} \\
& \left(\begin{array}{c}
\mu_{a, k+1} \\
\mu_{s, k+1}{ }^{\prime}
\end{array}\right)=\left(\begin{array}{c}
\mu_{a, k} \\
\mu_{s, k^{\prime}}
\end{array}\right)+\left(\begin{array}{c}
h_{a, k} \\
h_{s, k}
\end{array}\right) \\
& k=0,1,2,3, \ldots,
\end{aligned}
$$

where $k$ is an index and $h_{a}$ and $h_{s}$ are correction terms of $\mu_{a}$ and $\mu_{s}{ }^{\prime}$ The iterations were stopped when both $h_{a}$ and $h_{s}<1 \times 10^{-6}\left(\mathrm{~cm}^{-1}\right)$. With such strict criteria, previous research ${ }^{27}$ showed that the contribution of the Newton-Raphson method to the total prediction error was negligible.

Since in this study we want to extract two optical properties only, i.e., $\mu_{a}$ and $\mu_{s}{ }^{\prime}$, the MPR method implies exactly two input variables as well, i.e., $R\left(r_{1}\right)$ and $R\left(r_{2}\right)$. However, owing to tissue inhomogeneity and/or noisy measurement conditions, it might be advantageous to include more source-detector distances during measurements and then subsequently apply some sort of dimension-reduction method before the data are fed into the MPR method. To test such a procedure, we also applied principal component analysis (PCA) on the data from all six sourcedetector distances of the probe system and then used the resulting two main principal components $P_{1}$ and $P_{2}$ as input to the MPR method instead of $R\left(r_{1}\right)$ and $R\left(r_{2}\right)$.

All the prediction algorithms we applied in this paper were implemented in Matlab and run on a $166-\mathrm{MHz} \mathrm{PC}$. With this configuration, the prediction of a single set of $\mu_{a}$ and $\mu_{s}{ }^{\prime}$ could be performed in $\sim 60 \mathrm{~ms}$. 
Table 1. Optical Property Ranges of $6 \times 7$ Matrix of Intralipid-Ink Phantoms Determined from Integrating Sphere Measurements

\begin{tabular}{crc}
\hline & \multicolumn{2}{c}{ Optical Property Range $\left(\mathrm{cm}^{-1}\right)$} \\
\cline { 2 - 3 }$\lambda(\mathrm{nm})$ & \multicolumn{1}{c}{$\mu_{a}$} & $\mu_{s}{ }^{\prime}$ \\
\hline 660 & $0-0.36$ & $7.3-19.5$ \\
785 & $0-0.32$ & $6.1-16.3$ \\
805 & $0-0.31$ & $6.0-16.0$ \\
974 & $0.45-0.68$ & $4.8-12.7$ \\
\hline
\end{tabular}

\section{Phantoms}

Because of the unknown numerical apertures of the fiber probe light source and detectors, we chose to calibrate the system directly on a set of phantoms instead of using a mathematical light-propagation model. The phantoms consisted of well-defined aqueous solutions of Intralipid and black ink in cylindrical glass containers with a diameter of $10 \mathrm{~cm}$ and a height of $4 \mathrm{~cm}$. We determined the scattering and absorption spectra of the Intralipid and the black ink from integrating sphere ${ }^{27}$ and traditional transmission spectroscopy measurements. On the basis of these spectra, we mixed a $6 \times 7$ matrix of phantoms with $\mu_{a}$ and $\mu_{s}{ }^{\prime}$ ranges typical for skin tissue (see Table 1). The applied ranges of Intralipid concentrations were $0.6,0.8, \ldots, 1.6 \%$, and the range of the ink concentrations were $0.0,0.2, \ldots, 1.2 \%$. It should be noted that the absorption of pure ink is much higher than that of typical biologic substances; thus the ink concentrations in the following refers to a premixed basic ink-water solution with a biologically relevant absorption level. To perform the prediction experiments, we calibrated the probe system directly to the concentrations of the Intralipid and the ink in the phantoms, assuming that both the absorption of pure Intralipid ${ }^{29,30}$ and the scattering of the ink were negligible. At 660, 785, and $805 \mathrm{~nm}$ we assumed that the background absorption was negligible as well. However, water exhibits substantial absorption $^{31}$ at $974 \mathrm{~nm}\left(\mu_{a \text {,water }}=0.45 \mathrm{~cm}^{-1}\right)$. This was incorporated in the concentration-to-absorption tabulation by assuming a constant water absorption contribution at this particular wavelength (i.e., $c_{\text {ink }}=$ $0 \% \Rightarrow \mu_{a}=0.45 \mathrm{~cm}^{-1}$ ).

\section{Results}

Once the $6 \times 7$ matrix of phantoms was mixed, we determined the actual optical properties of a subset of the phantom matrix, using integrating sphere measurements. Figure 2(a) shows the mean absorption spectra of the six phantoms with $1 \%$ ink concentrations but varying Intralipid concentrations, and Fig. 2(b) shows the mean scattering spectra of the seven phantoms with $1 \%$ Intralipid concentrations but varying ink concentrations. We used the results from Fig. 2(a) to relate the concentrations of Intralipid and ink to the optical properties of the phantoms. The resulting optical property ranges of the $6 \times 7$ matrix phantoms are listed in Table 1 .

Figures 3(a) and 3(b) show the measured intensity
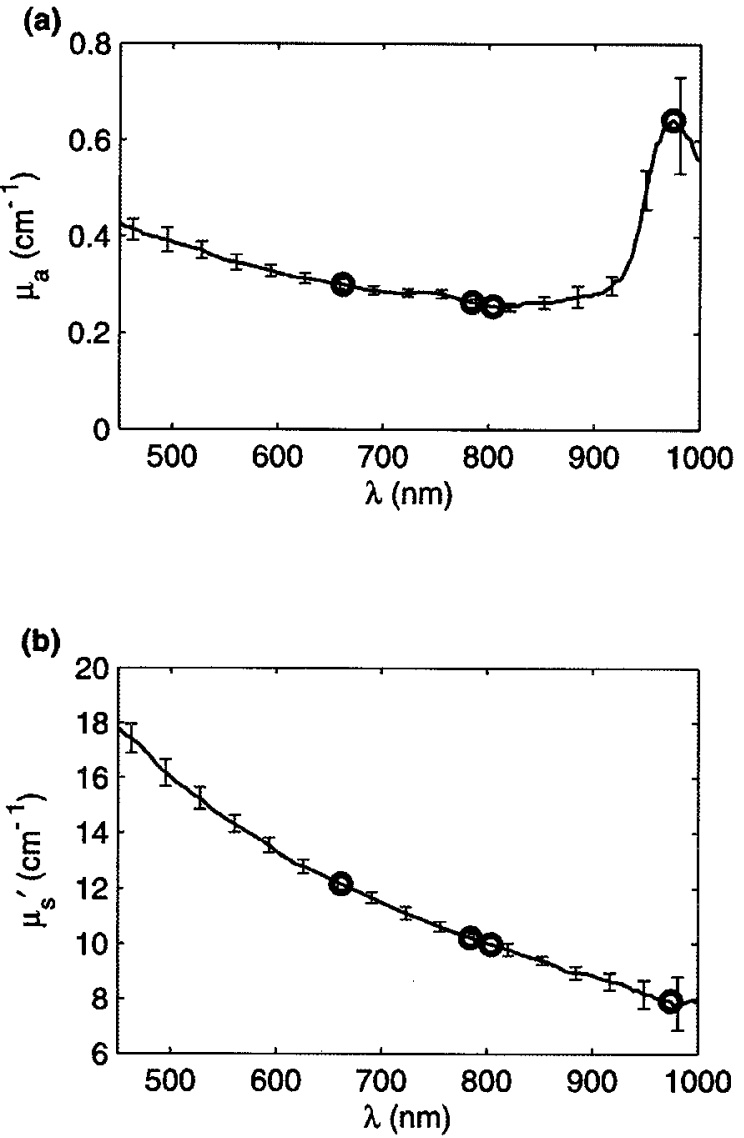

Fig. 2. Optical property spectra of the applied Intralipid-ink phantoms. (a) Mean of $\mu_{a}$ as a function of the wavelength $\lambda$ for the six phantoms with $1 \%$ ink concentration but varying Intralipid concentrations. (b) Mean of $\mu_{s}{ }^{\prime}$ as a function of the wavelength for the seven phantoms with $1 \%$ Intralipid concentration but varying ink concentrations. The error bars of the spectra in panels (a) and (b) show the standard deviations of $\mu_{a}$ and $\mu_{s}{ }^{\prime}$, respectively. The circles indicate the values at the four wavelengths of the probe. The two spectra were measured and calculated with an integrating sphere setup in conjunction with the MPR method.

$R(r)$ at $785 \mathrm{~nm}$ as a function of $\mu_{a}$ and $\mu_{s}{ }^{\prime}$ at $r=0.6$ $\mathrm{mm}$ and $r=7.8 \mathrm{~mm}$, respectively. Corresponding plots at the three remaining wavelengths of the probe, i.e., 660, 805, and $974 \mathrm{~nm}$, showed similar characteristics; i.e., they were also smooth and monotonic, which suggests that they may be fitted well by Eq. (1) with relatively low-order polynomials. Preliminary experiments showed that using Eq. (1) with $m=3$ provided the best overall calibration model, concerning accuracy and robustness. The prediction accuracy of the MPR method was tested with leaveone-out cross validation. This means that we successively performed predictions with the data from one phantom for prediction and the data from the remaining 41 phantoms of the $6 \times 7$ matrix for calibration. To ensure that all $R(r)$ variations were covered by the calibration models, we carried out predictions only on the $4 \times 5$ interior subset of the $6 \times$ 7 matrix. Table 2 presents the results from these cross-validation prediction tests. 
(a)

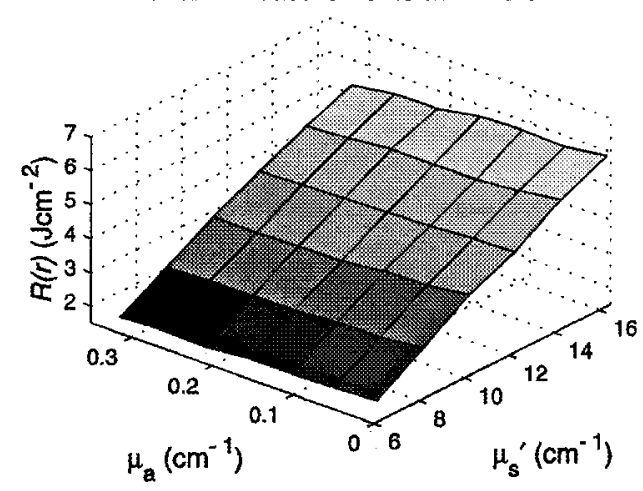

(c)

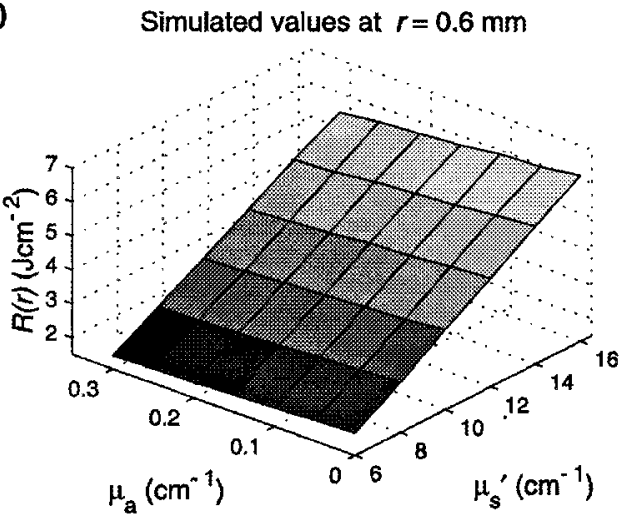

(b)

Phantom measurements at $r=7.8 \mathrm{~mm}$

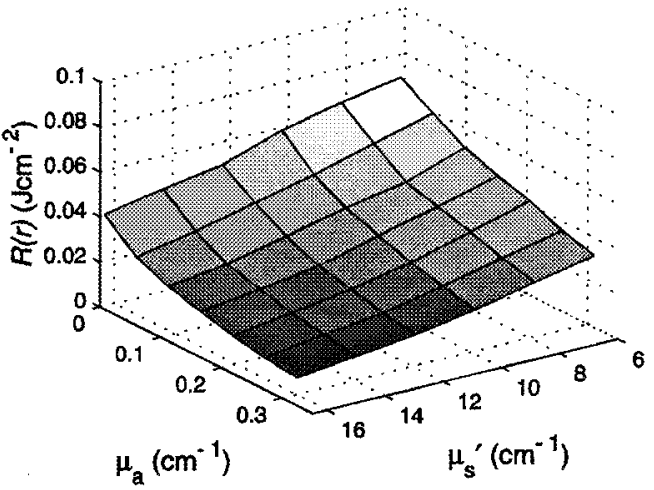

(d)

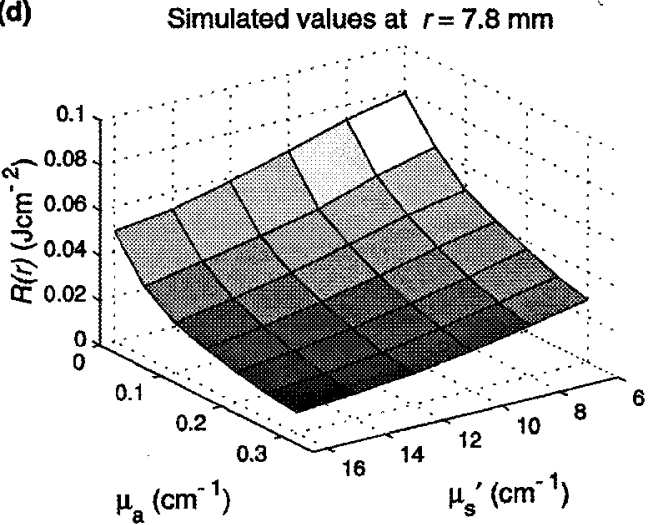

Fig. 3. Surface plots of $R(r)$ at $r_{1}=0.6 \mathrm{~mm}$ and $r_{2}=7.8 \mathrm{~mm}$ as a function of $\mu_{a}$ and $\mu_{s}{ }^{\prime}$. Panels (a) and (b) show the $R(r)$ plots of the $6 \times 7$ Intralipid phantoms at $785 \mathrm{~nm}$, and panels (c) and (d) show the corresponding $R(r)$ plots based on Monte Carlo simulations. Note that the arbitrary intensity units of measured $R(r)$ plots have been scaled in order to compare them with the simulated plots.

To check our experimental results, and to examine the robustness, noise sensitivity, and so on, of the applied calibration model and prediction algorithms, we also performed a series of numerical tests based on Monte Carlo simulations with a geometric configuration analogous to the fiber probe setup described in Subsection 3.A. However, unlike the measurements, the simulations were based on a collimated incident beam, a semi-infinite medium, and $1 / 2 \pi$ acceptance angle for collection of the diffuse reflectance. The tests were carried out as follows: (I) First, we generated a basic $6 \times 7$ calibration matrix with optical properties corresponding to the $6 \times 7$ phantom

Table 2. Leave-One-Out Cross-Validation Prediction Tests Based on Phantom Measurements ${ }^{a}$

\begin{tabular}{ccccc}
\hline & \multicolumn{4}{c}{ Prediction Errors (\%) } \\
\cline { 2 - 5 } & \multicolumn{4}{c}{$\mu_{a}$} \\
\hline$\lambda(\mathrm{nm})$ & Mean & Max & Mean & Max \\
600 & $3.3(2.5)$ & $18(6.7)$ & $1.7(1.7)$ & $3.7(4.0)$ \\
785 & 2.8 & 8.3 & 1.3 & 3.4 \\
805 & 2.6 & 9.0 & 1.5 & 4.2 \\
974 & 3.7 & 9.7 & 1.6 & 3.8 \\
\hline
\end{tabular}

${ }^{a}$ The errors are relative to the ranges of $\mu_{a}$ and $\mu_{s}{ }^{\prime}$ of the phantoms. The bracketed values at $\lambda=660 \mathrm{~nm}$ have been corrected for an outlier detected by visual inspection of the measured $R(r)$ data. matrix at $\lambda=785 \mathrm{~nm}$ and then performed prediction tests similar to the phantom cross-validation tests described above. Figure 4 shows a selected set of $R(r)$ profiles from these simulations [see also Figs. 3(c) and 3(d)]. (II) Next, to test the algorithms with independent calibration and prediction data, we generated a series of $20 R(r)$ data sets with random $\mu_{a}$ and $\mu_{s}{ }^{\prime}$ distributions and then performed predictions on this randomized set with a calibration model based on the full $6 \times 7$ basic matrix. (III) The basic $6 \times 7$ matrix was generated with $1 \times 10^{6}$ photons. To examine the impact of random measurement noise on the prediction performance, we generated a second $6 \times 7$ matrix with less noise by using $1 \times 10^{7}$ photons and performed identical cross-validation tests on this set. (IV) We also wanted to investigate the effect of using a calibration model with a higher $\mu_{a}$ and $\mu_{s}{ }^{\prime}$ resolution; thus we also carried out cross-validation tests on a $11 \times 13$ calibration matrix with the same $\mu_{a}$ and $\mu_{s}{ }^{\prime}$ range and number of photons as the basic $6 \times 7$ matrix. (V) Finally, we tested the effect of using PCA for dimension reduction as described in Subsection 3.B. The resulting mean and maximum prediction errors from the numerical tests $(\mathrm{I}-\mathrm{V}) \mathrm{de}-$ scribed above are summarized in Fig. 5.

To validate the optical property range of the applied calibration model, we performed a series of 

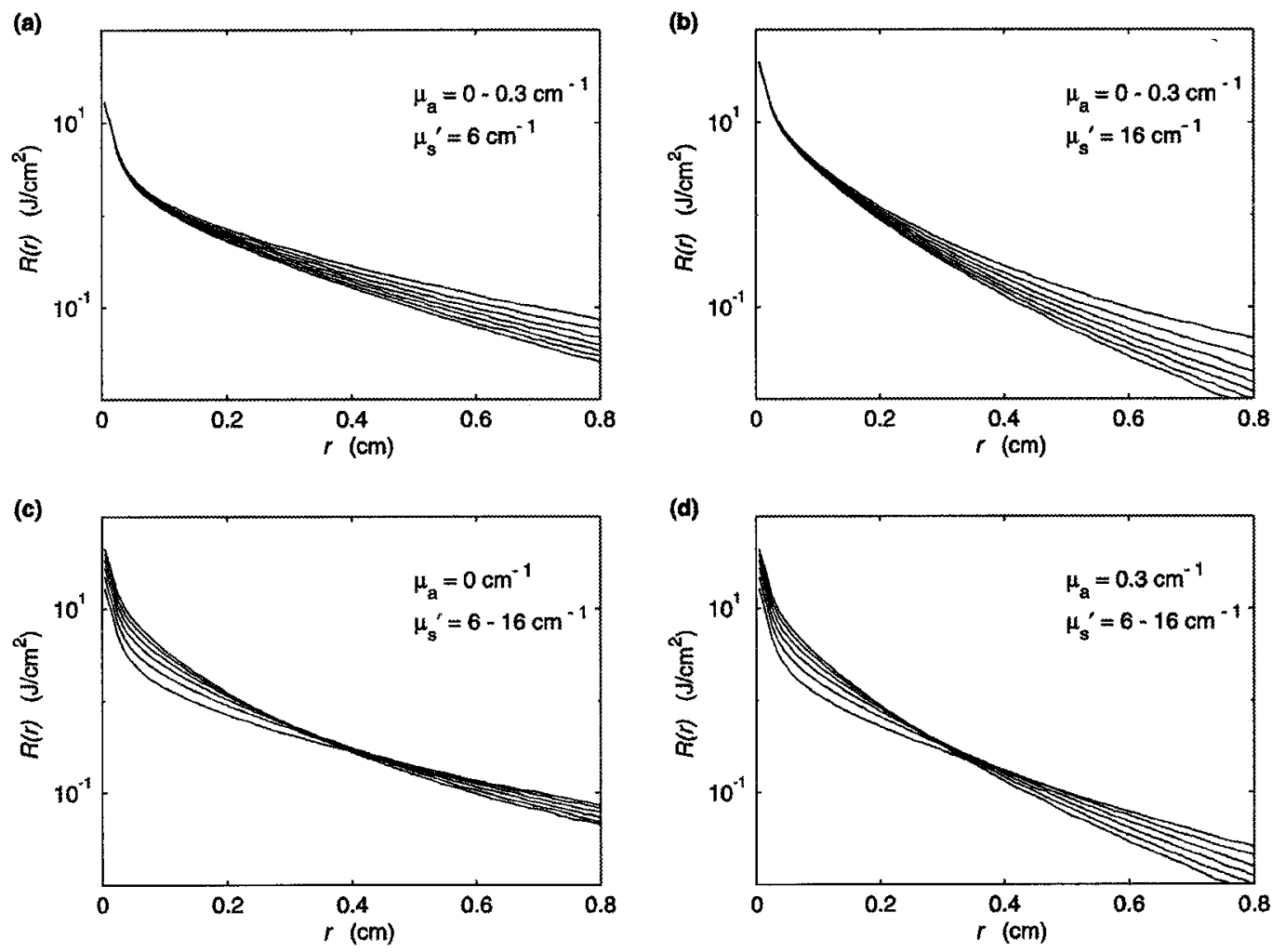

Fig. 4. Monte Carlo simulated $R(r)$ data for various combinations of $\mu_{a}$ and $\mu_{s}{ }^{\prime}$ within ranges typical for skin tissue at $785 \mathrm{~nm}$. In (a) and (b), $\mu_{s}{ }^{\prime}$ is kept constant at $6 \mathrm{~cm}^{-1}$ and $16 \mathrm{~cm}^{-1}$, respectively while $\mu_{a}$ is varied within the range $0-0.3 \mathrm{~cm}^{-1}$. In (c) and (d) $\mu_{a}$ is kept constant at $0 \mathrm{~cm}^{-1}$ and $0.3 \mathrm{~cm}^{-1}$, respectively, whereas $\mu_{s}{ }^{\prime}$ is varied within the range $6-16 \mathrm{~cm}^{-1}$.

probe measurements on the inner forearm of five healthy individuals. Figure 6 shows how the mean $R(r)$ of all five individuals (circles) at each probe distance is situated relative to the $R(r)$ ranges of the calibration model (vertical bars). Figure 6 also shows the predicted optical properties at all four wavelengths calculated from the mean $R(r)$ data with Eq. (1)-(3). It can be seen that the measured skin tissue at $\lambda=660 \mathrm{~nm}$ and $r=7.8 \mathrm{~mm}$ is outside the calibration model range, and therefore it is not possible to predict $\mu_{a}$ and $\mu_{s}{ }^{\prime}$ correctly in this case.

\section{Discussion}

\section{A. Geometry Considerations}

In Fig. 4(a) and 4(b) $\mu_{s}{ }^{\prime}$ is kept constant at values of 6 and $16 \mathrm{~cm}^{-1}$, respectively. In these two cases, it appears that changes in $\mu_{\alpha}$ have a negligible effect on $R(r)$ at distances close to the source. In Figs. 4(c) and $4(\mathrm{~d}), \mu_{a}$ is kept constant at values of 0 and 0.3 $\mathrm{cm}^{-1}$, respectively. Here it is notable that there is very little variation in $R(r)$ at $r \approx 0.35 \mathrm{~cm}$ in Fig. 4(c) and at $r \approx 0.3$ in Fig. 4(d). Using a figure of speech, we say that there is a pivot point in the $R(r)$ graphs at $r \approx 0.3 \mathrm{~cm}$, when $\mu_{a}$ is kept constant. The simulations in Figs. 4(a) and 4(b) indicates that $\mu_{s}{ }^{\prime}$ may be determined with good accuracy from close distance measurements only. To determine $\mu_{a}$ as well, Figs. $4(\mathrm{c})$ and 4(d) suggest that $R(r)$ measurements close to the pivot point should be included, since there is little variation in $R(r)$ as a function of $\mu_{s}^{\prime}$ at this point. Although, other authors ${ }^{32,33}$ support this argument, we based our experiments in this paper on close range distances in conjunction with distances well beyond the pivot point. We did this because our previous studies ${ }^{19}$ showed that this geometrical configuration provided a better accuracy than a configuration with close range distances in conjunction with distances near the pivot point.

\section{B. Phantom Measurements}

The prediction tests using Intralipid-ink phantoms show a good accuracy (see Table 2). The mean prediction errors at all four wavelengths is roughly 3\% for $\mu_{a}$, and $1.5 \%$ for $\mu_{s}{ }^{\prime}$, whereas the maximum prediction errors for $\mu_{a}$ and $\mu_{s}{ }^{\prime}$ are approximately $11 \%$ and $4 \%$, respectively. The prediction algorithm converged in all cases; however, the maximum error of $\mu_{a}$ at $\lambda=660 \mathrm{~nm}$ is remarkably high (18\%). A visual inspection of the raw $R(r)$ data at $660 \mathrm{~nm}$ revealed an outlier at $r=7.8 \mathrm{~mm}$ in one of the phantom measurements. This outlier could be due to an air bubble or a piece of dirt at one of the three 7.8-mm detectors in this particular measurement. When we removed the outlier from the calibration and prediction analysis at $\lambda=660 \mathrm{~nm}$, we obtained the improved results shown in brackets in Table 2. In general, the prediction errors of $\mu_{a}$ are approximately twice as high as the errors of $\mu_{s}{ }^{\prime}$. This may be attributed in part to the fact that $\mu_{a}$ is mainly deter- 
(a)

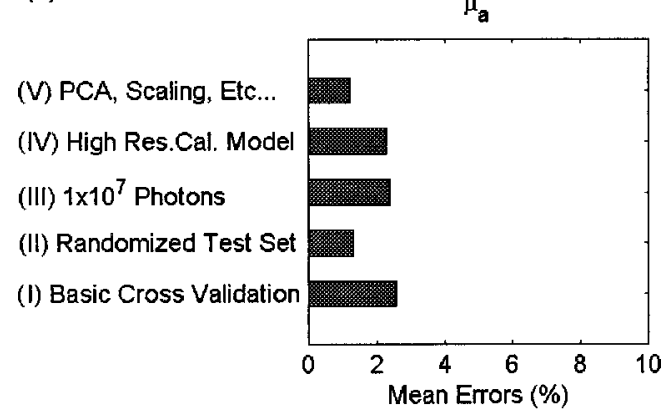

(c)

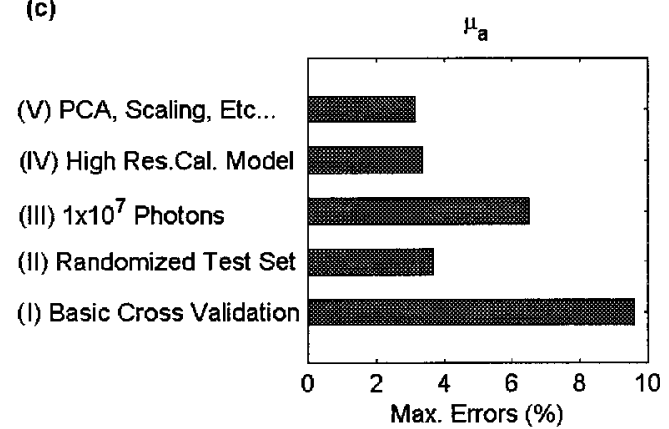

(b)

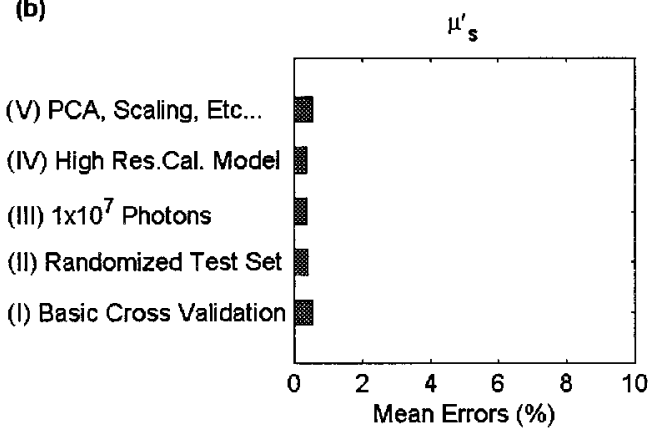

(d)

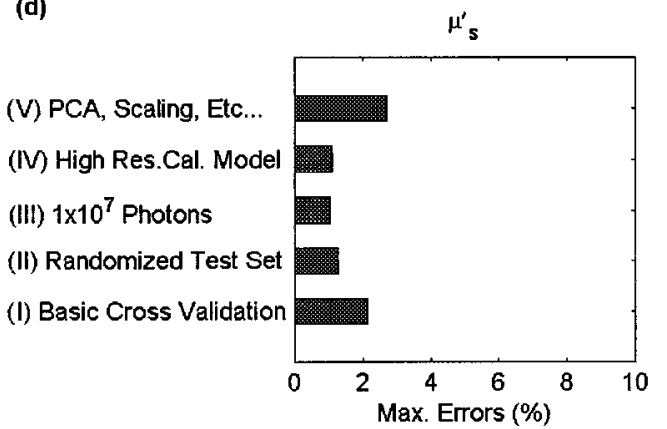

Fig. 5. Various numerical tests on Monte Carlo simulated $R(r)$ data. Panels (a) and (b) show the mean prediction errors of $\mu_{a}$ and $\mu_{s}{ }^{\prime}$, respectively. Panels (c) and (d) show the corresponding maximum prediction errors. From below: (I) cross validation on a basic $6 \times$ 7 calibration matrix ( $10^{6}$ photons) corresponding to phantom measurements at $785 \mathrm{~nm}$, (II) predictions on data with random $\mu_{a}$ and $\mu_{s}{ }^{\prime}$ distribution, (III) cross validation on $6 \times 7$ matrix generated with $1 \times 10^{7}$ photons, (IV) cross validation on a high-resolution $11 \times 13$ calibration matrix, (V) cross validation on the basic $6 \times 7$ calibration matrix with principal component analysis.

mined on the basis of the $R(r)$ data at $r=7.8 \mathrm{~mm}$, whereas $\mu_{s}^{\prime}$ is almost solely determined from $R(r)$ data at $r=0.6 \mathrm{~mm}$, where the signal level is $\sim 1000$ times the level at $r=7.8 \mathrm{~mm}$. The $\mu_{a}$ predictions are therefore more sensitive to any background noise interference during the measurements.

With reference to the comments on the pivot point in Subsection 5.A we also performed a series of prediction tests, in which we replaced the $R(r)$ probe data at $r_{2}=7.8 \mathrm{~mm}$ with probe data at the pivot point $r_{\text {pivot }}$ $=3.0 \mathrm{~mm}$. This had no significant effect on the $\mu_{s}{ }^{\prime}$ prediction errors, but both the mean and the maximum $\mu_{a}$ prediction errors increased $\sim 30 \%$ compared with results reported in Table 2.

\section{Numerical Tests}

The general shape of the measured and the simulated $R\left(\mu_{a}, \mu_{s}{ }^{\prime}\right)$ plots in Fig. 3 are very similar at corresponding detector distances. However, the simulated plots do show slightly higher $R\left(\mu_{a}, \mu_{s}{ }^{\prime}\right)$ levels for low $\mu_{a}$ and $\mu_{s}^{\prime}$ values at $r=7.8 \mathrm{~mm}$. This difference is severe enough to prevent the direct use of a calibration model based on Monte Carlo simulations for prediction analyses of $R(r)$ data collected with the fiber probe. The difference between the simulated and the measured $R(r)$ data in Figs. 3(b) and $3(\mathrm{~d})$ may be attributed to the different numerical apertures of the sources and the detectors in the two cases; e.g., the Monte Carlo simulations employ a collimated source beam, whereas the probe source beam is divergent.

The $\mu_{a}$ prediction errors of the simulated data shown in Figs. 5(a) and 5(c) are comparable with the errors of the measured data Table 2, whereas the $\mu_{s}{ }^{\prime}$ prediction errors of the simulated data [Figs. 5(b) and $5(d)]$ are lower than the errors of the measured data. In both cases the $\mu_{a}$ prediction errors are significantly higher than the $\mu_{s}{ }^{\prime}$ errors, probably owing to the noise sensitivity at $r=7.8 \mathrm{~mm}$ as discussed above. Figure 5 also shows a drop in the prediction errors when a randomized prediction data set is used. This may be because the quality of the calibration model fit during leave-one-out cross validation is reduced in the vicinity of the $\mu_{a}$ and $\mu_{s}{ }^{\prime}$ to be predicted.

From Fig. 5 it also appears that a reduction in measurement noise (i.e., more photons) or use of a calibration model with a higher $\mu_{a}$ and $\mu_{s}{ }^{\prime}$ resolution in both cases lead to a significant drop in maximum prediction errors, whereas the effect on the mean prediction errors is moderate. Finally, it can be seen from Fig. 5 that the use of PCA before calibration and prediction has a positive effect on the $\mu_{a}$ prediction errors, whereas the $\mu_{s}{ }^{\prime}$ prediction errors are slightly increased. In summary, the prediction accuracies obtained from calibration and prediction on phantoms are comparable with the accuracies obtained from calibration and prediction on Monte Carlo simulated data. The numerical tests suggest that the 
(a)
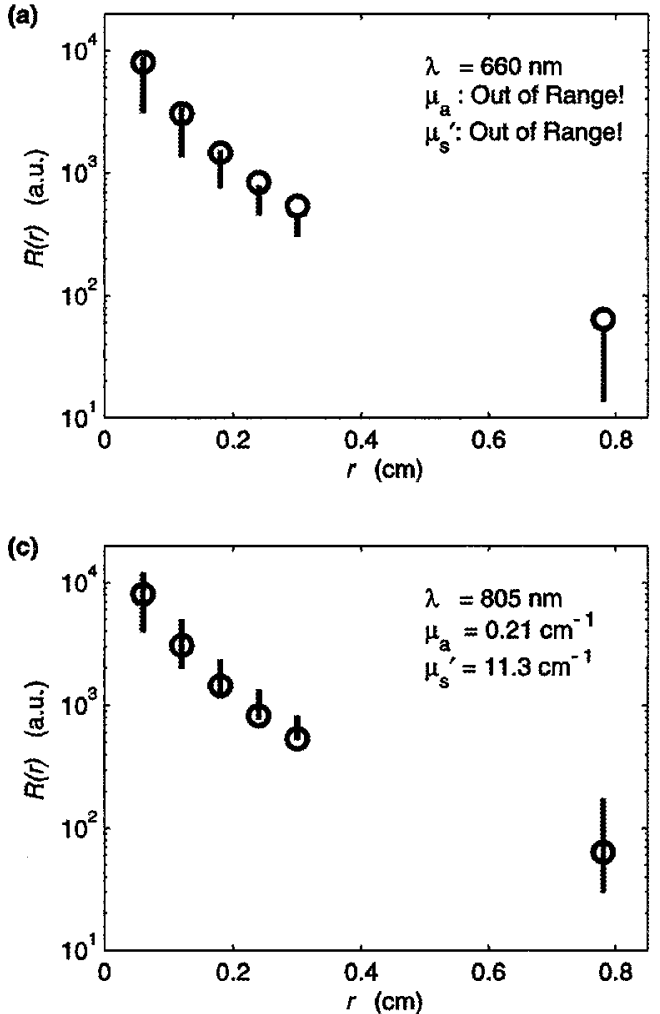

(b)

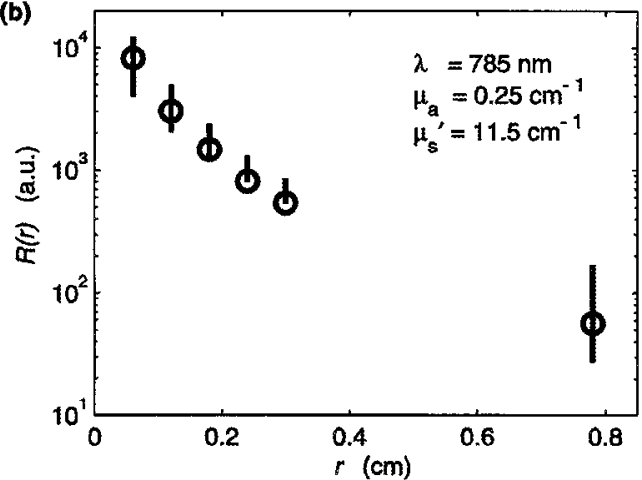

(d)

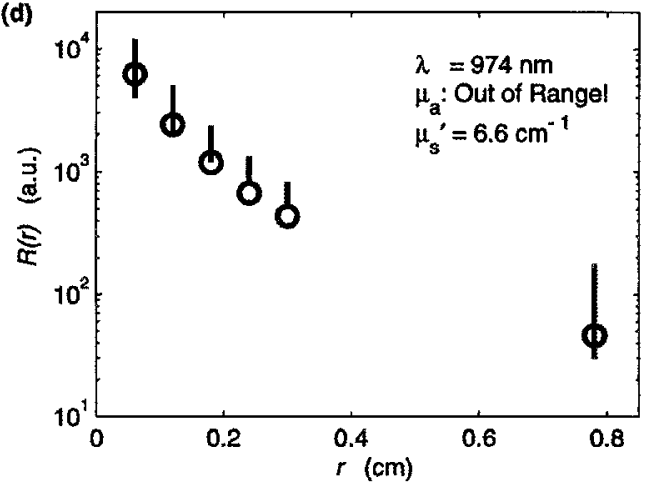

Fig. 6. Mean optical properties at all four wavelengths determined from $R(r)$ measurements on the forearm of five healthy individuals. The vertical bars indicate the valid range of the applied calibration model at various distances and wavelengths, and the circles indicate the mean measured $R(r)$ of the five individuals. At each of the four wavelengths, the stated values of $\mu_{a}$ and $\mu_{s}{ }^{\prime}$ were determined on the basis of the mean $R(r)$ values at $r_{1}=0.6 \mathrm{~mm}$ and $r_{2}=7.8 \mathrm{~mm}$.

prediction accuracy may be improved slightly by use of more samples during creation of the calibration model. However, the improvements may hardly justify the additional efforts of creating more calibration phantoms.

\section{Clinical Measurements}

From Fig. 6 it appears that the range of calibration model covers the span of the measured tissue $R(r)$ data in most cases, except at $\lambda=660 \mathrm{~nm}$. At this wavelength the mean of the measured $R(r)$ at $r_{2}=7.8$ $\mathrm{mm}$ is above the range of the calibration model. This suggests that either $\mu_{a}$ or $\mu_{s}^{\prime}$ (or both) of the skin tissue is lower than the minimum values of the calibration model. In the case of $\mu_{a}$ this is not possible, since the model range at $\lambda=660 \mathrm{~nm}$ is $0-0.36 \mathrm{~cm}^{-1}$. The minimum value of $\mu_{s}{ }^{\prime}$ of the model at this wavelength is $7.3 \mathrm{~cm}^{-1}$, and it is not likely that the measured $\mu_{s}{ }^{\prime}$ should be beneath this limit either. At the three remaining wavelengths the measured $R(r)$ at $r_{1}$ $=0.6 \mathrm{~mm}$ and $r_{2}=7.8 \mathrm{~mm}$ are within the limits of the calibration mode, and the extracted $\mu_{a}$ or $\mu_{s}{ }^{\prime}$ shows a reasonable correlation with other studies. ${ }^{20,34}$ However, at $974 \mathrm{~nm}$, it appears that the measured $R(r)$ data, at some of the intermediate distances (i.e., at $r=2.4$ and $3.0 \mathrm{~mm}$ ), are not within the ranges of the calibration model; i.e., the shape of the tissue $R(r)$ profiles are different from the shape of phantom profiles. There may be several reasons for this. First, in contrast to the phantoms, the volume of the skin tissue sampled by the probe is not homogeneous but basically consists of distinct layers, i.e., the stratum corneum, the vivid epidermis, the epidermis, and so on, Each of these layers has specific optical properties, and therefore the predicted values of $\mu_{a}$ or $\mu_{s}{ }^{\prime}$ in Fig. 6 are a compound of the optical properties of each separate layer. Second, the ratio of the refractive indices between the probe and the sample $\left(n_{\text {probe }} /\right.$ $\left.n_{\text {sample }}\right)$ is different in the two cases; i.e., $n_{\text {tissue }} \approx 1.40$ (Ref. 35) and $n_{\text {phantom }} \approx 1.33$. Finally, the probetissue interface may not have been optimal, which in turn may lead to light piping effects between the probe and the stratum corneum. Such effects may cause an increase of the farther $R(r)$ values relative to the values close to the light source, which also might explain the out-of-range problems encountered at $\lambda=$ $660 \mathrm{~nm}$. To summarize, it is essential to ensure a proper optical contact and apply a uniform and reproducible mechanical pressure in order to obtain valid predictions from contact probe measurements.

\section{E. Acquisition and Analysis Speed}

As stated in Subsection 3.A, the maximum acquisition rate of the fiber probe system for all four wavelengths is $\sim 100 \mathrm{~Hz}$, which is sufficient for analyzing the optical property dynamics of most physiological systems. The current software implementation of the prediction algorithms leads to an analysis time 
for one set of $\mu_{a}$ or $\mu_{s}{ }^{\prime}$ of $\sim 50 \mathrm{~ms}$, i.e., an analysis rate of $5 \mathrm{~Hz}$ when all four wavelengths are included. To match the analysis rate to the acquisition rate of the probe system, the speed of the prediction algorithms may be increased substantially by application of a more contemporary PC and/or by compiling the algorithms in, e.g., the $\mathrm{C}$ programming language.

\section{Conclusions}

We have demonstrated a versatile, fast, and accurate probe system for real-time noninvasive determination of tissue optical properties from continuous wave (cw) spatially resolved diffuse reflectance measurements. The current calibration of the system was intended to match a typical range of absorption and reduced scattering coefficients of skin tissue, and preliminary clinical trials on a set of healthy individuals showed a good consistency except at $\lambda=660 \mathrm{~nm}$. At this wavelength the measured tissue data were outside the range of the calibration model in some instances. This problem may be solved by extension of the calibration model range, but the problem may also originate in the fact that the system was calibrated on homogeneous one-layer phantoms, whereas skin tissue is an inhomogeneous multilayer structure. Furthermore, the clinical trials clearly demonstrated that it is imperative to ensure proper and reproducible refractive-index matching and mechanical contact properties to obtain valid results. All things considered, the system and the method we have presented here provide a sound basis for future development of compact and dedicated systems for noninvasive or minimally invasive medical diagnostics and monitoring. Still, further research is required for exploring the applicability for specific biomedical implementations.

The authors acknowledge financial support from the Danish Academy of Technical Sciences.

\section{References}

1. J. Welch, M. J. C. van Gemert, M. W. Star, and B. C. Wilson, "Overview of tissue optics," Optical-Thermal Response of Laser-Irradiated Tissue, A. J. Welch and M. J. C. van Gemert, eds. (Plenum, New York, 1995), Chap. 2.

2. J. W. Feather, D. J. Ellis, and G. Leslie, "A portable reflectometer for the rapid quantification of cutaneous haemoglobin and melanin," Phys. Med. Biol. 33, 711-722 (1988).

3. S. L. Jacques, "Reflectance spectroscopy with optical fiber devices, and transcutaneous bilirubinometers," in Biomedical Optical Instrumentation and Laser-Assisted Biotechnology, A. M. Verga Scheggi, S. Martellucci, A. N. Chester, and R. Pratesi, eds., Vol. E325 of NATO ASI Series (Kluwer Academic, Dordrecht, The Netherlands, 1996), pp. 83-94.

4. M. A. Franceschini, E. Gratton, and S. Fantini, "Noninvasive optical method of measuring tissue and arterial saturation: an application to absolute pulse oximetry of the brain," Opt. Lett. 24, 829-831 (1999).

5. S. L. Jacques, "Origins of tissue optical properties in the UVA, Visible, and NIR regions," in Advances in Optical Imaging and Photon Migration, R. R. Alfano and J. G. Fujimoto, eds., Vol. 2 of OSA Topics in Optics and Photonics Series (Optical Society of America, Washington, D.C., 1996), 364-369.

6. A. H. Hielscher, J. R. Mourant, and I. J. Bigio, "Influence of particle size and concentration on the diffuse backscattering of polarized light from tissue phantoms and biological cell suspensions," Appl. Opt. 36, 125-135 (1997).

7. J. R. Mourant, J. P. Freyer, A. H. Hielscher, A. A. Eick, D. Shen, and T. M. Johnson, "Mechanisms of light scattering from biological cells relevant to noninvasive optical-tissue diagnostics," Appl. Opt. 37, 3586-3593 (1998).

8. J. T. Bruulsema, J. E. Hayward, T J. Farrell, M. S. Patterson, L. Heinemann, M. Berger, T. Koschinsky, C. J. Sandahl, H. Orskov, M. Essenpreis, R. G. Schmelzeisen, and D. Bocker, "Correlation between blood glucose concentration in diabetics and noninvasively measured tissue optical scattering coefficient," Opt. Lett. 22, 190-192 (1997).

9. M. S. Patterson, B. Chance, and B. C. Wilson, "Time resolved reflectance and transmittance for the non-invasive measurement of tissue optical properties," Appl. Opt. 28, 2331-2336 (1989).

10. S. Andersson-Engels, R. Berg, A. Persson, and S. Svanberg, "Multispectral tissue characterization with time-resolved detection of diffusely scattered white light," Opt. Lett. 18, 16971699 (1993).

11. B. Chance, M. Cope, E. Gratton, N. Ramanujam, and B. Tromberg, "Phase measurement of light absorption and scatter in human tissue," Rev. Sci. Instrum. 69, 3457-3481 (1998).

12. S. Fantini, M. A. Franceschini, J. S. Maier, S. A. Walker, B. Barbieri, and E. Gratton, "Frequency-domain multichannel optical detector for noninvasive tissue spectroscopy and oximetry," Opt. Eng. 34, 32-42 (1995).

13. T. J. Farrell, M. S. Patterson, and B. Wilson, "A diffusion theory model of spatially resolved, steady-state diffuse reflectance for noninvasive determination of tissue optical properties in vivo," Med. Phys. 19, 879-888 (1992).

14. P. Marquet, F. Bevilacqua, C. Depeursinge, and E. B. DeHaller, "Determination of reduced scattering and absorption coefficients by a single charge-coupled-device array measurement. I. Comparison between experiments and simulations," Opt. Eng. 34, 2055-2063 (1995).

15. A. Kienle, L. Lilge, M. S. Patterson, R. Hibst, R. Steiner, and B. C. Wilson, "Spatially resolved absolute diffuse reflectance measurements for noninvasive determination of the optical scattering and absorption coefficients of biological tissue," Appl. Opt. 35, 2304-2314 (1996).

16. R. Bays, G. Wagnieres, D. Robert, D. Braichotte, J. F. Savary, P. Monnier, and H. van den Bergh, "Clinical determination of tissue optical properties by endoscopic spatially resolved reflectometry," Appl. Opt. 35, 1756-1766 (1996).

17. J. R. Mourant, T. Fuselier, J. Boyer, T. M. Johnson, and I. J. Bigio, "Predictions and measurements of scattering and absorption over broad wavelength ranges in tissue phantoms," Appl. Opt. 36, 949-957 (1997).

18. M. G. Nichols, E. L. Hull, and T. H. Foster, "Design and testing of a white-light, steady-state diffuse reflectance spectrometer for determination of optical properties of highly scattering systems," Appl. Opt. 36, 93-104 (1997).

19. J. S. Dam, P. E. Andersen, T. Dalgaard, and P. E. Fabricius, "Determination of tissue optical properties from diffuse reflectance profiles by multivariate calibration," Appl. Opt. 37, 772778 (1998).

20. R. M. Doornbos, L. Lang, R. Alders, F. W. Cross, and H. J. C. M. Sterenborg, "The determination of in vivo human tissue optical properties and absolute chromophore concentrations using spatially resolved steady-state diffuse reflectance spectroscopy," Phys. Med. Biol. 44, 967-981 (1999).

21. T. H. Pham, F. Bevilacqua, T. Spott, J. S. Dam, B. Tromberg, and S. Andersson-Engels, "Quantifying the absorption and reduced scattering coefficients of tissuelike turbid media over a broad spectral range with a noncontact Fourier transform hyperspectral imaging," Appl. Opt. 39, 6487-6497. 
22. L. V. Wang, "Source of error in calculation of optical diffuse reflectance from turbid media using diffusion theory," Comput. Methods Program Biomed. 61, 163-170 (2000).

23. L. H. Wang, S. L. Jacques, and L. Q. Zheng, "MCML-Monte Carlo modeling of photon transport in multi-layered tissues," Comput. Methods Program Biomed. 47, 131-146 (1995).

24. T. J. Farrell, B. C. Wilson, and M. S. Patterson, "The use of a neural network to determine tissue optical properties from spatially resolved diffuse reflectance measurements," Phys. Med. Biol. 37, 2281-2286 (1992).

25. H. C. van de Hulst, Multiple Light Scattering (Academic, New York, 1980), Vols. I and II.

26. R. Graff, J. G. Aarnoudse, F. F. M. de MulHenk, and W. Jentink, "Similarity relations for anisotropic scattering in absorbing media," Opt. Eng. 32, 244-252 (1993).

27. J. S. Dam, T. Dalgaard, P. E. Fabricius, and S. AnderssonEngels, "Multiple polynomial regression method for determination of biomedical optical properties from integrating sphere measurements," Appl. Opt. 39, 1202-1209 (2000).

28. W. F. Cheong and A. J. Welch, "A review of the optical properties of biological tissue," IEEE J. Quantum Electron. 26, 2166-2185 (1990).

29. H. J. van Staveren, C. J. M. Moes, J. van Marle, S. A. Prahl, and M. J. C. van Gemert, "Light scattering in Intralipid-10\% in the wavelength range of 400-1100 nm, " Appl. Opt. 30, 45074514 (1991).

30. S. T. Flock, S. L. Jacques, B. C. Wilson, W. M. Star, and M. J. C. van Gemert, "Optical properties of Intralipid: a phantom medium for light propagation studies, " Lasers Surg. Med. 12, 510-519 (1992).

31. S. J. Matcher, M. Cope, and D. T. Delpy, "Use of the water absorption spectrum to quantify tissue chromophore concentration changes in near-infrared spectroscopy," Phys. Med. Biol. 39, 177-196 (1994).

32. J. R. Mourant, I. J. Bigio, D. A. Jack, T. M. Johnson, and H. D. Miller, "Measuring absorption coefficients in small volumes of highly scattering media: source-detector separations for which path lengths do not depend on scattering properties," Appl. Opt. 36, 5655-5661 (1997).

33. G. Kumar, "Optimal probe geometry for near-infrared spectroscopy of biological tissue," Appl. Opt. 36, 2286-2293 (1997).

34. C. R. Simpson, M. Kohl, M. Essenpreis, and M. Cope, "Nearinfrared optical properties of ex vivo human skin and subcutaneous tissues measured using the Monte Carlo inversion technique," Phys. Med. Biol. 43, 2465-2478 (1998).

35. F. P. Bolin, L. E. Preuss, R. C. Taylor, and R. J. Ference, "Refractive index of some mammalian tissues," Appl. Opt. 28, 2297-2303 (1989). 